\title{
№. 67-895
}

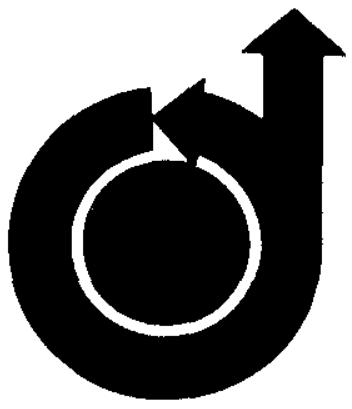

\section{SURFACES FOR COMPUTER-AIDED AIRCRAFT DESIGN}

by

STEVEN A. COONS

Massachusetts Institute of Technology

Cambridge, Massachusetts

and

BERTRAM HERZOG

University of Michigan

Ann Arbor, Michigan

AIAA Paper

No. 67-895

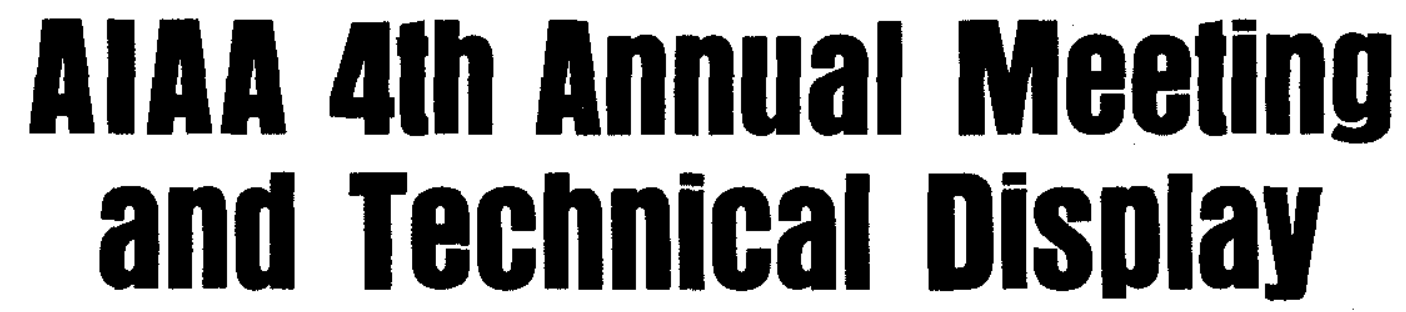

ANAHEIM, CALIFORNIA/OCTOBER 23-27, 1967

First publication rights reserved by American Institute of Aeronautics and Astronautics, 1290 Avenue of the Americas, New York, N. Y, 10019. Abstracts may be published without permission if credit is given to author and to AlAA. (Price-AlAA Member $\$ 1.00$, Nonmember $\$ 1.50$ ) 


\section{Abstract}

A simple but general way is described to define free-form surfaces such as airplane fuselages, wings, fillets, ducts, and other shapes by means of man-machine graphical interaction with a computer. In the past, much attention has been directed toward fitting mathematical functions to surfaces already defined by a mesh of points. The present discussion will center around the philosophy that in the preliminary phase of shape description the computer's aid should be enlisted at the very beginning, and that in this way the results of preliminary surface design become the first "master dimen sions" of the airplane directly, without the necessity of refairing or other subsequent troatment. Furthermore, the computer data structure for the description of shape also serves as the skeleton upon which other associated data can be hung, such as velocity fields, pressures, temperatures, forces, and other physical quantities that arise in connection with analytical and design procedures.

\section{Introduction}

Free-form surfaces play a central role in the design of airplanes, automobiles, ships, and many machine parts, such as dif. ferential housings, forgings, castings, and telephone hand-sets.

Traditionally, mathematical representations of such surfaces have attempted to fit mathematical equations to selected points on already designed surfaces; that is, surfaces that were designed originally by some graphical process. This paper describes a technique for representing surface points mathematically at the initial stage of the design process. The advantages of such a technique are sufficiently obvious as to require no further elaboration at this point.

\section{Surfaces}

- With a light-pen and CRT device, the designer can delineate a few important curves describing a shape he has in mind. The surface defined by these curves is exhibited on the display console, where the designer can observe immediately the results of his actions. If the characteristics of the resulting surface are not satisfactory, he can modify the original curves; or he can add other curves in regions previously implicit, thereby making his wishes explicit to the computer. When curves are added or modified, the computer then alters the previous surface so that the new surface passes smoothly through the new curves until the desired shape is achieved. The mathematical description of this shape is retained by the computer and can be used to produce full-sized drawings, to direct fabricating machinery (e.g., numerically controlled milling machines), to sink forming dies, produce foundry patterns, or to carve out full-sized models.

Points are represented as matrices (or vectors) such as $\left[\begin{array}{lll}x & y & z\end{array}\right]$. Any set of three ordered numbers represents a point in space.

For a curve, the coordinates of the variable point that sweeps out the curve can be represented by three functions of a single independent variable, called a parameter. Thus

$$
\begin{aligned}
& x=x(u) \\
& y=y(u) \\
& z=z(u) .
\end{aligned}
$$

Such a representation has advantages over explicit forms like

$$
\begin{aligned}
& y=f(x) \\
& z=g(x),
\end{aligned}
$$

since in the 1atter case the variable $x$ plays a somewhat different role from that played by $y$ and $z$. In some sense, this is undesirable. It is particularly awkward when, for example, the slope $d y / d x$ of a curve becomes infinite. The parametric form also has advantages over the implicit form

+ This rescarch was supported in part by Bell Telephone Laboratories, Inc.; the Advanced Research Projects Agency, Department of Defense; and the Office of Naval Research.

* Associate Professor, Department of Mechanical Engineering, Massachusetts Institute of Technology; and Research Associate in Computer Graphics, The Aiken Computation Laboratory, Harvard University.

** Associate Professor, Department of Industrial Engineering, The University of Michigan. 


$$
\begin{aligned}
& f(x, y, z)=0 \\
& g(x, y, z)=0,
\end{aligned}
$$

because it is very difficult to compute points on the curve from these simultaneous relationships. The parametric form of curve description is virtually standard in studies of curves and surfaces in differential geometry. A curve is the locus of a point that moves in space with a single degree of freedom. Similarly, a surface is the locus of a point that moves in space with two degrees of freedom.

When we represent a surface parametrical1y, we write

$$
\begin{aligned}
& x=x(s, t) \\
& y=y(s, t) \\
& z=z(s, t) .
\end{aligned}
$$

These represent three functions of the two independent variables, or parameters, s and $t$. We can write the vector equation

$$
\left[\begin{array}{lll}
x & y & z
\end{array}\right]=[x(s, t) y(s, t) z(s, t)] \text {. }
$$

Now the three functions of the variables as they appear on the right-hand side can be represented by the single symbol (st). This is a "bi-literal" symbol that stands for the vector consisting of the three functions. It is a convenient, compact symbol that saves a great deal of notation.

We plan to restrict our attention to surface regions bounded by four arbitrary space curves. If, additionally, for computational simplicity, we restrict the range of the variables $s$ and $t$ to lie between 0 and 1 , or, in symbols

$$
0 \leqslant s, t \leqslant 1 \text {, }
$$

then we have a symbol to represent a general point on a surface (st), or a boundary curve where $s$ has been set to its lower limit zero ( $0 t$ ), and similarly for the three other boundaries:

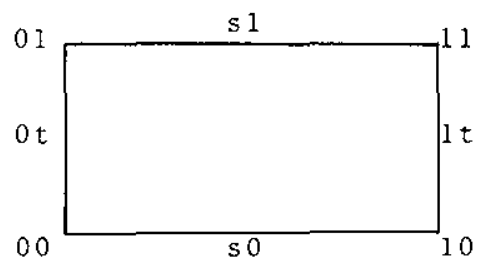

Thus, all four boundaries represent functions of two variables, but with one of the variables held temporarily fixed. If both variables are held fixed, say for example if $s=1$ and $t=0$, the biliteral symbol becomes (10), and we are then talking about one of the corners of the patch.
Later we shall introduce some additional notational conventions, but for the timc being these will serve to enable us to write some equations.

We shall use two functions, $F_{0}$ and $F_{1}$, which have the property that ${ }^{\circ}$

$$
F_{0}(0)=1 \quad F_{1}(1)=1
$$

and

$$
F_{0}(1)=0 \quad F_{1}(0)=0 .
$$

The actual form of these functions is cntirely arbitrary, so long as these endconditions are fulfilled. Their form has no effect on the validity of the surface equation. We will refer to these functions as "blending functions."

With this notation, the surface equation can be written in matrix form:

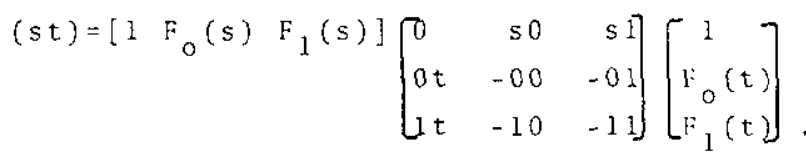

The entries in the $3 \times 3$ matrix consist of the four axbitrarily chosen boundary curves, so, si, $0 t$, and $1 \mathrm{t}$, together with the corner points 00 , 01 , 10 , and 11 .

It should be remembered that so is really a shorthand expression for the three vector components of the curve; it represents the vector

$$
\mathrm{s} 0=[\mathrm{x}(\mathrm{s} 0) \mathrm{y}(\mathrm{s} 0) \mathrm{z}(\mathrm{s} 0)],
$$

where each coordinate component represents a suitably chosen function of the singlo paramcter $s$; the $t$ parameter in this case is held fixed and equal to 0 . Similar remarks apply to the other three boundary curves.

If, in addition to the previously stated requirements on the $F$ functions, we also state that for

$$
\begin{aligned}
& \frac{d F_{0}(s)}{d s}=F_{0}^{\prime}(s) \\
& F_{0}^{\prime}(0)=0 \\
& F_{0}^{\prime}(1)=0 \\
& F_{1}^{\prime}(0)=0
\end{aligned}
$$

and

$$
F_{j}^{\prime}(1)=0 \text {, }
$$

then the surface has the peculiar property of having tangent vectors across boundaries that depend only upon the tangent vectors at corners. For example, using the notation

$$
s 0_{t}=\left.\frac{\partial(s t)}{\partial t}\right|_{t=0}
$$


we can write, after differentiating the equation and setting $t=0$,

$$
\begin{aligned}
s 0_{t} & =\left[\begin{array}{lll}
1 & F_{0}(s) & F_{1}(s)
\end{array}\right]\left[\begin{array}{ccc}
0 & 0 & 0 \\
00 & 0 & 0 \\
10 & 0 & 0
\end{array}\right]\left[\begin{array}{c}
1 \\
F_{0}(0) \\
F_{1}(0)
\end{array}\right] \\
& +\left[\begin{array}{lll}
1 & F_{0}(s) F_{1}(s)
\end{array}\right]\left[\begin{array}{ccc}
0 & s 0 & s 1 \\
00 & -00 & -01 \\
10 & -10 & -11
\end{array}\right]\left[\begin{array}{c}
0 \\
F_{0}^{1}(0) \\
F_{1}^{1}(0)
\end{array}\right]
\end{aligned}
$$

When we introduce the conditions of the F functions, the previous equation becomes

$$
s 0_{t}=00_{t} F_{0}(s)+10_{t} F_{1}(s) \text {. }
$$

This shows that the tangent vector across so in the $t$ direction depends only upon $00 \mathrm{t}$ and $10 \mathrm{t}$, the tangent vectors at the ends of the ${ }^{\mathrm{s}} 0$ curve, in the $t$ direction; it does not depend upon curve shape, nor upon the shape of the other boundaries, except at 00 and 20 .

Hence, two patches will have continuous slope across a shared boundary. Furthermore, if the second derivatives of the F func.. tions are zero for

$$
\begin{aligned}
& s=0 \\
& s=1,
\end{aligned}
$$

then two adjacent patches will be curvaturo. continuous across a boundary, provided their boundary curves are curvaturencontinuous. We can escalate the order of continuity between two patches simply by adding conditions on the $F$ functions.

An analogous equation can be constructed for the modification of this primary surface, in case its intrinsic boundary slope does not match that of some adjacent sur. face. Such mismatch can occur only in casc the adjacent surface cannot be described by the primary surface equation. In theory at least, many surfaces indeed do not lend themselves to such description. Consequert ly if one of the surface patches is to adjoin such a surface and be slope-continuous with it, the boundary slope of the surface patch must be modified.

The equation for such modification is

$$
(s t)=\left[\begin{array}{llll}
1 & G_{0}(s) & G_{1}(s)
\end{array}\right]\left[\begin{array}{ccc}
0 & s 0 & s l_{t} \\
0 t_{s} & -00_{s t} & -01 s_{s t} \\
1 t_{s} & -100_{s t} & -11 s_{s t}
\end{array}\right]\left[\begin{array}{c}
1 \\
G_{0}(t) \\
G_{1}(t)
\end{array}\right]
$$

It obviously bears a strong resemblance to the form of the primitive surface equation. The $G$ functions have the properties

$$
G_{0}(0)=G_{0}(1)=G_{1}(0)=G_{1}(1)=0
$$

and

$$
\begin{array}{ll}
G_{0}^{\prime}(0)=1 & G_{0}^{\prime}(1)=0 \\
G_{1}^{\prime}(1)=1 & G_{1}^{1}(0)=0 .
\end{array}
$$

With these properties, the surface described has null vectors for all boundaries, but has tangent vectors across boundaries as shown in the matrix: i.e., so, slt, $0 t_{s}$, and $1 t_{s}$. Since these can be $a \stackrel{r}{\text {. }}$. bitrary vector functions of $s$ and $t$, any two surfaces can be joined together so as to maintain slope continuity across their common boundaries.

\section{Boundary Curves}

Traditionally, two kinds of curves have been used in airplane lines design: cubic polynomials and conics. Unfortunately, each of these curve-forms by itself has certain drawbacks, In the parametric form, for ordinary cubics, the entire shape of a curve segment is governed by end tangent vectors. Sometimes these end tangent vectors lead to unwanted hooks and bulges in the curve segments. On the other hand, conics, although more benignly behaved, cannot by their very nature yield curves with points of inflection. Yet such curves very often exist in aircraft shapes, for example, in wing fillets.

Because of these shortcomings, a new curve type has been developed. It is based upon rational polynomial functions. It contains both conics and ordinary cubics as special cases, and provides a great degree of generality and flexibility.

We start be establishing the form of the function. Let $v$ be a vector, so that, for example,

$$
v=\left[\begin{array}{llll}
x & y & z & 1
\end{array}\right]
$$

or

$$
v=\left[\begin{array}{lll}
x & y & 1
\end{array}\right]
$$

or

$$
v=\left[\begin{array}{ll}
x & 1
\end{array}\right] .
$$

The fixst of these can be thought of as the vector (or matrix) of coordinates on a space curve; the second is the vector of coordinates for a plane curve, and the last is the vector of a single varying coordinate of a curve. Since the last vector yields the most general case, we shall begin with it, and show how one might evaluate a set of numbers in a matrix to define each of the parametric coordinates of a curve.

The product of $v$ and a variable scalar w is

$$
w V=\left[\begin{array}{ll}
w x & w
\end{array}\right] .
$$

Here, both $w x$ and $w$ are cubic functions of a parameter $u$, and obviously

$$
x=\frac{w x}{w} \text {. }
$$


This is the ratio of two cubic polynomials, hence the term rational function.

We can represent the two cubic polynomials by the matric equation

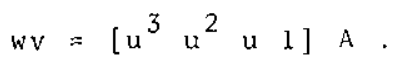

Since

$$
w V=\left[\begin{array}{ll}
w x & w
\end{array}\right],
$$

the matric A must consist of four rows and two columns of constant coefficients.

We now proceed to show how these numbers may be found so as to define a coordinate of a curve. We shall be interested in the end-point coordinates of the curve at

$$
\mathrm{u}=0
$$

and

$$
\mathrm{u}=1 \text {. }
$$

These coordinates are

$$
v_{0}=\left[x_{0} 1\right]
$$

and

$$
v_{1}=\left[\begin{array}{ll}
x_{1} & 1
\end{array}\right]
$$

respectively. A tangent vector anywhere on the curve is clearly

$$
v^{\prime}=\left[x^{\prime} 0\right] \text {, }
$$

where the prime mark means differentiation with respect to the parameter $u$. The tangent vectors at $u=0$ and $u=1$ are therefore

$$
v_{0}^{1}=\left[x_{0}^{1} 0\right]
$$

and

$$
v_{1}^{\prime}=\left[x_{1}^{\prime} 0\right]
$$

respectively.

Now, differentiating both sides of

$$
w v=\left[u^{3} u^{2} u r\right] A \text {, }
$$

we obtain

$$
(w v)^{\prime}=\left[\begin{array}{llll}
3 u^{2} & 2 u & 1 & 0
\end{array}\right] \mathrm{A} .
$$

Substitution of $u=0$ and $u=1$ into these two expressions yields

$$
\left[\begin{array}{c}
w_{0} v_{0} \\
w_{1} v_{1} \\
\left(w_{0} v_{0}\right)^{\prime} \\
\left(w_{1} v_{1}\right)^{\prime}
\end{array}\right]=\left[\begin{array}{llll}
0 & 0 & 0 & 1 \\
1 & 1 & 1 & 1 \\
0 & 0 & 1 & 0 \\
3 & 2 & 1 & 0
\end{array}\right] \mathrm{A} .
$$

The $4 \times 4$ matrix on the right has an inverse, and we may write

$$
\begin{aligned}
A & =\left[\begin{array}{llll}
0 & 0 & 0 & 1 \\
1 & 1 & 1 & 1 \\
0 & 0 & 1 & 0 \\
3 & 2 & 1 & 0
\end{array}\right]^{-1}\left[\begin{array}{c}
w_{0} v_{0} \\
w_{1} v_{1} \\
\left(w_{0} v_{0}\right) \\
\left(w_{1} v_{1}\right)
\end{array}\right] \\
& =\left[\begin{array}{cccc}
2 & -2 & 1 & 1 \\
-3 & 3 & -2 & -1 \\
0 & 0 & 1 & 0 \\
1 & 0 & 0 & 0
\end{array}\right]\left[\begin{array}{l}
w_{0} v_{0} \\
w_{1} v_{1} \\
w_{0}^{\prime} v_{0}+w_{0} v_{0}^{\prime} \\
w_{1}^{\prime} v_{1}+w_{1} v_{1}^{1}
\end{array}\right] .
\end{aligned}
$$

The square matrix inverse is constant and always the same and reappears in the algebra so often that we shall henceforth call it the matrix $M$.

The matric equation can be factored and rewritten in the form

$$
A=M\left[\begin{array}{cccc}
w_{0} & 0 & 0 & 0 \\
0 & w_{1} & 0 & 0 \\
w_{0}^{\prime} & 0 & w_{0} & 0 \\
0 & w_{1}^{\prime} & 0 & w_{1}
\end{array}\right]\left[\begin{array}{c}
v_{0} \\
v_{1} \\
v_{0}^{\prime} \\
v_{1}^{1}
\end{array}\right]
$$

The right-hand matrix of $v^{\prime} s$ represents the desired end-conditions on the curve. In our present case, it is of course a $4 \times 2$ matrix.

The middle matrix is $4 \times 4$ and contains the four numbers [ $w$ w $\left.w^{1} w^{\prime}\right]$. Any arbitrary set of four humbers inserted into this matrix will serve to define a unique pair of cubic functions of the param eter $u$, from which $x$ can be found by using, as we have said, the ratio

$$
x=\frac{w x}{w} \text {. }
$$

Rather than pick these four numbers arbitrarily, we shall impose sufficient further conditions on the curve to define $\left[w_{0} w_{1} w_{0}^{1} w_{1}^{1}\right]$ uniquely.

We begin by introducing desired second derivative vectors at the end points. These vectors are, clearly,

$$
v_{0}^{\prime \prime}=\left[x_{0}^{\prime \prime} 0\right]
$$

and

$$
v_{1}^{\prime \prime}=\left[\begin{array}{ll}
x_{1}^{\prime \prime} & 0
\end{array}\right] \text {. }
$$

Incidentally, for the vectors

$$
\begin{aligned}
& v^{\prime}=\left[\begin{array}{lll}
x^{\prime} & y^{\prime} & 0
\end{array}\right] \\
& v^{\prime \prime}=\left[\begin{array}{lll}
x^{\prime \prime} & y^{\prime \prime} & 0
\end{array}\right],
\end{aligned}
$$

if the determinant of the matrix 


$$
\left[\begin{array}{ll}
x^{\prime} & y^{\prime} \\
x^{\prime \prime} & y^{\prime \prime}
\end{array}\right]
$$

vanishes, the curve will have a point of inflection at $v$. If the determinant is positive, the center of curvature will lie on the left as one proceeds along the curve; if the detcrminant is negative, the center of curvature will lie on the right. If two curve segments have equal $v^{\prime}$ and $v^{\prime \prime}$ at a junction, they are continuous both in slope and curvature at such a junction.

When we take second derivatives of both sides of

$$
w v=\left[\begin{array}{llll}
u^{3} & u^{2} & u & 1
\end{array}\right] A,
$$

we obtain

$$
(w v)^{\prime \prime}=\left[\begin{array}{llll}
6 u & 2 & 0 & 0
\end{array}\right] \mathrm{A} \text {. }
$$

At $u=0$ this is

$$
\left(w_{0} v_{0}\right)^{\prime \prime}=\left[\begin{array}{llll}
0 & 2 & 0 & 0
\end{array}\right] M\left[\begin{array}{l}
w_{0} v_{0} \\
w_{1} v_{1} \\
w_{0}^{\prime} v_{0}+w_{0} v_{0}^{\prime} \\
w_{1}^{1} v_{1}+w_{1} v_{1}^{1}
\end{array}\right] \text {. }
$$

But

$$
\begin{aligned}
& \left(w_{0} v_{0}\right)^{\prime \prime}=w_{0}^{\prime \prime} v_{0}+2 w_{0}^{1} v_{0}^{1}+w_{0} v_{0}^{\prime \prime}, \\
& \text { and solving for } w_{0} v_{0}^{\prime \prime}, \\
& w_{0} v_{0}^{\prime \prime}=\left(w_{0} v_{0}\right)^{\prime \prime}-w_{0}^{\prime \prime} v_{0}-2 w_{0}^{\prime} v_{0}^{\prime} .
\end{aligned}
$$

Now

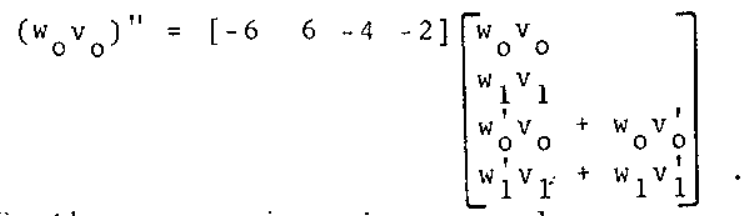

Furthermore, since in general

$$
(w v)^{\prime \prime}=\left[(w x)^{\prime \prime} w^{\prime \prime}\right] \text {, }
$$

the quantity $w^{\prime \prime}$ is the second component of the vector of (wv)" and therefore is associated with the last column of the inatrix

$$
\left[\begin{array}{l}
w_{0} v_{0} \\
w_{1} v_{1} \\
w_{0}^{1} v_{0}+w_{0} v_{0}^{\prime} \\
w_{1}^{1} v_{1}+w_{1} v_{1}^{1}
\end{array}\right]
$$

But the last components of $v_{0}$ and $v_{1}$ are both one, and the last components of 1 $v_{0}^{\prime}$ and $v_{1}^{\prime}$ are both zero.
Hence

$$
w_{0}^{\prime \prime}=\left[\begin{array}{llll}
-6 & 6 & -4 & -2
\end{array}\right]\left[\begin{array}{c}
w_{0} \\
w_{1} \\
w_{0}^{\prime} \\
w_{1}^{\prime}
\end{array}\right] .
$$

By combining results, we can now write:

$$
\begin{aligned}
& w_{0} v_{0}^{\prime \prime}=\left[\begin{array}{llll}
-6 & 6 & -4 & -2
\end{array}\right]\left[\begin{array}{l}
w_{0}\left(v_{0}-v_{0}\right) \\
w_{1}\left(v_{1}-v_{0}\right) \\
w_{0}^{\prime}\left(v_{0}-v_{0}\right)+w_{0} v_{0}^{\prime} \\
w_{1}^{\prime}\left(v_{1}-v_{0}\right)+w_{1} v_{1}^{1}
\end{array}\right]-2 w_{0}^{1} v_{0}^{\prime} \\
& =6 w_{1}\left(v_{1}-v_{0}\right)-4 w_{0} v_{0}^{1}-2 w_{1}^{1}\left(v_{1}-v_{0}\right) \\
& -2 w_{1} v_{1}^{1}-2 w_{0}^{1} v_{0}^{1} \text {. }
\end{aligned}
$$

Collecting

$$
\begin{aligned}
w_{0} v_{0}^{\prime \prime}=w_{0}\left(-4 v_{0}^{\prime}\right) & +w_{1}\left(6\left(v_{1}-v_{0}\right)-2 v_{1}^{\prime}\right) \\
& +w_{0}^{\prime}\left(-2 v_{0}^{\prime}\right)+w_{1}^{\prime}\left(-2\left(v_{I}-v_{0}\right)\right) .
\end{aligned}
$$

We now restore this last expression to matrix form:

$$
w_{0} v_{0}^{\prime \prime}=\left[w_{0} w_{1} w_{0}^{1} w_{1}^{\prime}\right]\left[\begin{array}{c}
-4 v_{0}^{1} \\
6\left(v_{1}-v_{0}\right)-2 v_{1}^{\prime} \\
-2 v_{0}^{\prime} \\
-2\left(v_{1}-v_{0}\right)
\end{array}\right] \text {. }
$$

In our present case, the matrix on the right consists of a column of numbers and a column of zeros. Hence the column of zeros can be discarded, and the result is a $4 \times 1$ matrix. On the left, $w_{0} v_{0}^{\prime \prime}$ is a scalax.

Similarly, we can find by analogous algebraic procedures that

$$
w_{1} v_{1}^{\prime \prime}=\left[w_{0} w_{1} w_{0}^{\prime} w_{1}^{\prime}\right]\left[\begin{array}{c}
6\left(v_{0}-v_{1}\right)+2 v_{0}^{1} \\
4 v_{1}^{1} \\
2\left(v_{0}-v_{1}\right) \\
-2 v_{1}^{1}
\end{array}\right] \text {. }
$$

Then, writing a matric equation, we have so far

$$
\left[w_{0} v_{0}^{\prime \prime} w_{1} v_{1}^{\prime \prime}\right]=\left[\begin{array}{ll}
w_{0} & w_{1} \\
w_{0}^{\prime} & w_{1}^{\prime}
\end{array}\right][P \quad Q],
$$

where [P Q] represents a $4 \times 2$ matrix consisting of the separate $4 \times 1$ matrices for $w_{0} v_{0}^{\prime \prime}$ and $w_{1} v_{1}^{\prime \prime}$, written side by side as columis.

We now introduce another condition, Let it be required that the curve pass through the point 


$$
v_{c}=\left[\begin{array}{ll}
x_{c} & 1
\end{array}\right],
$$

when

$$
u=1 / 2,
$$

where this value of $u$ is of course arbitrary. This condition leads to

$$
v_{c}=(1 / 8)\left[\begin{array}{llll}
1 & 2 & 4 & 8
\end{array}\right] M\left[\begin{array}{l}
w_{0} v_{0} \\
w_{1}^{\prime} v_{1} \\
w_{0}^{\prime} v_{0}+w_{0} v_{0}^{\prime} \\
w_{1}^{\prime} v_{1}+w_{1} v_{1}^{\prime}
\end{array}\right] .
$$

By algebraic manipulations similar to the preceding, we can rearrange the equation to read

$$
8 v_{c}=\left[w_{0} w_{1} \cdot w_{0}^{1} w_{1}^{1}\right]\left[\begin{array}{c}
4 v_{0}+v_{0}^{\prime} \\
4 v_{1}-v_{1}^{\prime} \\
v_{0} \\
-v_{1}
\end{array}\right] .
$$

In this equation, $v$ has two components, $x_{c}$ and $l$. The right ${ }^{c}$ hand matrix is a $4 \times 2$. Call it the $R$ matrix. Then we can adjoin these matrices to obtain:

$$
\left[\begin{array}{lll}
w_{0} v_{0}^{\prime \prime} & w_{1} v_{1}^{\prime \prime} & 8 v_{c}
\end{array}\right]=\left[\begin{array}{llll}
w_{0} & w_{1} & w_{0}^{\prime} & w_{1}^{\prime}
\end{array}\right]\left[\begin{array}{lll}
P & Q & R
\end{array}\right] \text {. }
$$

Now [P $Q$ R] represents a $4 \times 4$ matrix; $P$ and $Q$ are each $4 \times 1$ matrices, but $R$ is a $4 \times 2$ matrix. We next transfer $w_{0} v_{0}^{\prime}$ and $w_{1} v_{1}^{\prime \prime}$ to the right-hand side, obtaining

$\left[\begin{array}{llll}0 & 0 & 8 v_{c}\end{array}\right]=\left[\begin{array}{lll}w_{0} & w_{1} & w_{0}^{\prime} \\ & w_{I}^{\prime}\end{array}\right]\left(\left[\begin{array}{lll}P & Q & R\end{array}\right]-\left[\begin{array}{cccc}v_{0}^{\prime \prime} & 0 & 0 & 0 \\ 0 & v_{1}^{\prime \prime} & 0 & 0 \\ 0 & 0 & 0 & 0 \\ 0 & 0 & 0 & 0\end{array}\right]\right)$

The right-hand matrix is now a $4 \times 4$. Provided its determinant does not vanish, it has an inverse, and

$$
\left[\begin{array}{llll}
w_{0} & w_{1} & w_{0}^{\prime} & w_{1}^{\prime}
\end{array}\right]=\left[\begin{array}{lll}
0 & 0 & 8 v_{c}
\end{array}\right] S \text {, }
$$

where $S$ is the $4 \times 4$ inverse of the matrix.

Now that the components of

$$
\left[\begin{array}{llll}
w_{0} & w_{1} & w_{0}^{\prime} & w_{1}^{\prime}
\end{array}\right]
$$

have been evaluated, the curve is complete ly defined, since the rational function (wv) / $w$ is completely defined.

With some loss of generality and flexibility, we can have

$$
v=\left[\begin{array}{lll}
x & y & 1
\end{array}\right]
$$

a plane curve. We shail next show that for an appropriate choice of the components of

$$
\left[\begin{array}{llll}
w_{0} & w_{1} & w_{0}^{1} & w_{i}^{1}
\end{array}\right] \text {, }
$$

the curve reduces to a conic.

We have the equation

$$
\begin{aligned}
w V & =\left[\begin{array}{lll}
w x & w y & w
\end{array}\right] \\
& =\left[\begin{array}{llll}
u^{3} & u^{2} & u & 1
\end{array}\right] A .
\end{aligned}
$$

In this case, $A$ is a $4 \times 3$ matrix. Now if the top row of this matrix is $\left[\begin{array}{lll}0 & 0 & 0\end{array}\right]$, the equation reduces to

$$
w v=\left[\begin{array}{llll}
u^{2} & u & 1
\end{array}\right] \mathrm{A}
$$

when the top row of A has been omitted. $A$ is now a $3 \times 3$ matrix, and it is possible to show that this equation is a para. metric form for the general conic, expressed as a quadratic rational function.

Without going into details of derivation, the conditions on the components of

$$
\left[\begin{array}{llll}
w_{0} & w_{1} & w_{0}^{1} & w_{1}^{\prime}
\end{array}\right]
$$

that make the top row of A vanish are embedded in the matric equation:

$$
\left[\begin{array}{llll}
w_{0} & w_{1} & w_{0}^{1} & w_{1}^{1}
\end{array}\right]=\left[\begin{array}{llll}
0 & 0 & 0 & w_{0}
\end{array}\right]\left[\begin{array}{ccc}
2 v_{0}+v_{0}^{1} & 1 \\
-2 v_{1}+v_{1}^{1} & 0 \\
v_{0} & 0 \\
v_{1} & 0
\end{array}\right]^{-1}
$$

The square matrix on the right has an inverse in case the determinant

$$
\left|\begin{array}{c}
-2 v_{1}+v_{1}^{\prime} \\
v_{0} \\
v_{1}
\end{array}\right| \neq 0
$$

It is always possible in all of the foregoing to set

$$
w_{0}=1
$$

This is because al 1 equations are homogeneous. It is never possible for

$$
w_{0}=0 \text {, }
$$

since this leads to certain degenerate cases.

We remark in passing that when

$$
\left[\begin{array}{lll}
w_{0} & w_{1} & w_{0}^{\prime} \\
w_{1}^{\prime}
\end{array}\right]=\left[\begin{array}{llll}
1 & 1 & 0 & 0
\end{array}\right] \text {, }
$$

the equation reduces to the ordinary para- 
metric cubic, given by

$$
v=\left[\begin{array}{llll}
u^{3} & u^{2} & u & 1
\end{array}\right] \quad M\left[\begin{array}{c}
v_{0} \\
v_{1} \\
v_{0}^{\prime} \\
0 \\
v_{1}
\end{array}\right]
$$

and $w$ is constant and equal to one.

Hence the rational polynomial functions contain, as special cases, a 11 conics, ordinary cubics, and of course therefore straight lines and circles. Their use for boundary curves for surface patches is obvious. They maintain tangent vector continuity between adjacent patches; indecd, if the $F$ and $F$ functions are constructed as rational functions, we can establish the $F_{1}$ function

$$
F_{1}(u)=\frac{u^{3}}{3 u^{2}-3 u+1} \text {. }
$$

This function has the end conditions

$$
\left[\begin{array}{l}
v_{0} \\
v_{1} \\
v_{0}^{\prime} \\
v_{1}^{\prime} \\
v^{\prime \prime} \\
0 \\
v_{1}^{\prime \prime}
\end{array}\right]=\left[\begin{array}{lll}
0 & 0 & 1 \\
1 & 1 & 1 \\
1 & 0 & 0 \\
1 & 0 & 0 \\
0 & 0 & 0 \\
0 & 0 & 0
\end{array}\right] .
$$

Since

$$
\begin{aligned}
& v_{0}^{\prime \prime}=\lambda v_{0}^{\prime}=0\left[\begin{array}{lll}
1 & 0 & 0
\end{array}\right] \\
& v_{1}^{\prime \prime}=\lambda v_{1}^{\prime}=0\left[\begin{array}{lll}
1 & 0 & 0
\end{array}\right],
\end{aligned}
$$

the curve has a point of inflection at $u=0$ and $u=1$. Hence its use ensures curvature continuity across boundaries between patches, provided, of course, the boundary curves have similar curvature continuity at patch corners. can put

Ihe curve is symmetric. Furthexmore, we

$$
F_{0}(u)=1-F_{1}(u)
$$

and obtain dixectly the $F$ function, another cubic rational funetion, whth similar properties to $F_{1}$.

\section{Example}

We shall work out the equation for the $F_{l}(u)$ blending function with the customary stipulations that

$$
F_{1}(1)=1, F_{1}(0)=F_{1}^{\prime}(0)=F_{1}^{\prime}(1)=0 \text {, }
$$

and with the two additional stipulations that

$$
F_{1}^{\prime \prime}(0)=\mathrm{P}_{1}^{\prime \prime}(1)=0 \text {. }
$$

This blending function will give both slope and curvature continuity across the common boundary between two contiguous patches. The end conditions are, for

$$
v=\left[F_{0}(u) \quad 1\right]
$$

$$
\left[\begin{array}{l}
v_{0} \\
v_{1} \\
v_{0}^{\prime} \\
v_{1}^{\prime} \\
v_{0}^{\prime \prime} \\
v_{1}^{\prime \prime}
\end{array}\right]=\left[\begin{array}{l}
01 \\
11 \\
00 \\
00 \\
00 \\
00
\end{array}\right]
$$

The matrix

$$
\left[\begin{array}{lll}
P & Q & R
\end{array}\right]-\left[\begin{array}{cccc}
v_{0}^{\prime \prime} & 0 & 0 & 0 \\
0 & v_{1}^{\prime \prime} & 0 & 0 \\
0 & 0 & 0 & 0 \\
0 & 0 & 0 & 0
\end{array}\right]=\left[\begin{array}{cccc}
0 & -6 & 0 & 4 \\
6 & 0 & 4 & 4 \\
0 & -2 & 0 & 1 \\
-2 & 0 & -1 & -1
\end{array}\right]
$$

obtained by direct substitution in the given form. Its inverse is

$$
\left[\begin{array}{rrrr}
0 & -1 & 0 & -4 \\
1 & 0 & -4 & 0 \\
-2 & 2 & 6 & 6 \\
2 & 0 & -6 & 0
\end{array}\right]=S
$$

Now set $v_{c}=\left[\begin{array}{ll}1 / 2 & 1\end{array}\right]$ for symmetry. Then since $u=c 1 / 2$,

$$
\begin{aligned}
& {\left[\begin{array}{llll}
w_{0} & w_{1} & w_{0}^{1} & w_{1}^{1}
\end{array}\right]=\left[\begin{array}{lll}
0 & 0 & 8 v_{c}
\end{array}\right] S} \\
& =\left[\begin{array}{llll}
0 & 0 & 4 & 8
\end{array}\right]\left[\begin{array}{rrrr}
0 & -1 & 0 & -4 \\
1 & 0 & -4 & 0 \\
-2 & 2 & 6 & 6 \\
2 & 0 & -6 & 0
\end{array}\right]
\end{aligned}
$$$$
=\left[\begin{array}{llll}
8 & 8 & -24 & 24
\end{array}\right] \text {. }
$$

Now if $w_{0}=1$, instead of 8 , the cquation becomes

$$
\left[\begin{array}{llll}
w_{0} & w_{1} & w_{0}^{1} & w_{1}^{1}
\end{array}\right]=\left[\begin{array}{llll}
1 & 1 & -3 & 3
\end{array}\right] .
$$


We have

$$
A=M\left[\begin{array}{l}
w_{0} v_{0} \\
w_{1} v_{1} \\
w_{0}^{1} v_{0}+w_{0} v_{0}^{\prime} \\
w_{1}^{\prime} v_{1}+w_{1} v_{1}^{1}
\end{array}\right]
$$

and substituting the values of

$$
\left[\begin{array}{llll}
w_{0} & w_{1} & w_{0}^{1} & w_{1}^{1}
\end{array}\right] \text { : }
$$

$A=\left[\begin{array}{rrrr}2 & -2 & 1 & 1 \\ 3 & 3 & -2 & -1 \\ 0 & 0 & 1 & 0 \\ 1 & 0 & 0 & 0\end{array}\right]\left[\begin{array}{rr}0 & 1 \\ 1 & 1 \\ 0 & -3 \\ 3 & 3\end{array}\right]=\left[\begin{array}{rr}1 & 0 \\ 0 & 3 \\ 0 & -3 \\ 0 & 1\end{array}\right]$.

Final $1 \mathrm{y}$

$$
\begin{aligned}
& w v=\left[\begin{array}{lll}
w x & w
\end{array}\right]=\left[\begin{array}{ll}
u^{3} & u^{2} u
\end{array}\right]\left[\begin{array}{rr}
1 & 0 \\
0 & 3 \\
0 & -3 \\
0 & 1
\end{array}\right] \\
& {\left[\begin{array}{ll}
w x & w
\end{array}\right]=\left[\begin{array}{ll}
u^{3} & 3 u^{2}-3 u+1
\end{array}\right]}
\end{aligned}
$$

Hence

$$
x=\frac{w x}{w}=\frac{u^{3}}{3 u^{2}-3 u+1}=F_{1}(u)
$$

as required. The other $F_{0}$ function is

$$
\begin{aligned}
F_{0}(u) & =1-F_{1}(u)=1-\frac{u^{3}}{3 u^{2}-3 u+1} \\
& =\frac{-u^{3}+3 u^{2}-3 u+1}{3 u^{2}-3 u+1} .
\end{aligned}
$$

This is seen to be a rational cubic function also.

\section{Bibliography}

1. Coons, S.A., "Surfaces for Computeraided Design of Space Figures," Electronic Systems Laboratory Memo 9442-M-139, Massachusetts Institute of Technology, Cambridge, Mass.; also in Project MAC Memo, MAC-M-253, Massachusetts Institute of Technology, Cambridge, Mass.

2. Ferguson, J.C., "MuItivariable Curve Interpolations," Boeing Document D2. 22504; also in proceedings of the ACM, 1964, pp. 121 et seq.

3. Kelly, J.P., and South, N.E., "Analytic Surface Methods," Numerical Control Development Unit, Ford Motor Co., Dearborn, Michigan, December 1965.
4. Johnson, W.L., "Analytic Surfaces for Computer-aided Design," SAE Convention, 1966, Paper 660152 .

5. llamilton, M.L., and Weiss, A.D., "An Approach to Computer-aided Preliminary Ship Design," Electronic Systems laboratoy, TM-228, Massachusetts Institute of Technology, Cambridge, Mass., January 1965 .

6. De Iotto, I., and Galimberti, R., "Innovative Design with Computer Graphics," Alta Frequenza, Vol. 36, No. 5, 1967. 